CANALES WEB EN CIENCIAS

\section{DE LA COMUNICACIÓN}

\section{Producción multimedia}

para la docencia

e investigación en

Documentación

\section{informativa}

\section{Alfonso López Yepes}

\section{Catedrático de Documentación}

Facultad de Ciencias de la Información. Universidad Complutense de Madrid. Avd. Complutense s/n, 28043 Madrid (España) - Email: alopezeccinf.ucm.es

\section{Resumen}

La creación inicial en una primera fase de un portal de portales de documentación informativa (e-Doculnfo http://multidoc.rediris.es /edocuinfo/) que integra varios portales y posibilita asimismo el acceso a un canal temático de documentación vía internet (TVDoc Complumedia-RTVDoc http://complumedia.ucm.es/canal.php?p1= $\underline{\text { rtvdoc }})$, juntamente con el funcionamiento de otros dos $(E-T V$ http://www.ucm.es/info/e-tv y Cine@Tele Online canal vídeo http://www.cineytele.com/vervideo.php? $=_{\mathrm{w}}$ G1wya 3D30) tienen como objetivo la difusión de contenidos y la generación de una
Palabras clave

Canal web, ciencias de la información, cine@tele online,complumedia-rtvdoc, documentación informativa, e-docuinfo, e-tv, multimedia bajo demanda, producción multimedia, servicio de documentación multimedia, tvdoc

Key Words

Channel web, information science, cine@tele online, complumedia-rtvdoc, information research, e-docuinfo, e-tv, multimedia on demand, multimedia production, multimedia information service, tvdocs

\section{Abstract}

The article describes the structure of contents and setup of the, already

fully operative in 2009, RTVDoc: radio-television web channel and online-mediateca, which is integrated in the documentation multimedia service (Multidoc) of the faculty of communication sciences of the university

Complutense of Madrid. This thematic channel produces, canalizes and spreads contents in a distributed and collaborative way. Shaped as online university platform, real time and "on demand", the channel is the materialization of the idea of creation and publication materials for teachers, students, researchers and professionals of mass media. The wide variety of multimedia didactic materials are targeted to the educational and professionals sectors these contents can be found subdivided in the newspaper library, music library, photo library and video library categories of the channel. 
programación en el ámbito documental de las ciencias de la información, de forma distribuida y colaborativa, en estrecha relación con los parámetros de la web 2.0 o web social, y de la Universidad-Empresa. Una segunda fase ha supuesto la producción de numerosos contenidos sistematizados por materias y el establecimiento de verdaderos canales IPTV de emisión total (en consecuencia, TVDoc evoluciona a ComplumediaRTVDoc), y que a su vez plantean la antesala de una tercera fase evolutiva, el establecimiento de una Red iberoamericana de documentación multimedia, ya en ciernes (http://multidoc.rediris.es/infodocnet). Estos canales temáticos, que pueden emitir información en tiempo real o en diferido -vídeo bajo demanda-, vehiculan información procedente -en el caso de e-Docuinfo- de la hemeroteca, fonoteca, fototeca, videoteca, en fin, de la mediateca del portal a través de un servidor de streaming. La existencia del portal de portales y de los mencionados canales temáticos web se benefician asimismo, por sus mayores posibilidades de difusión informativa, de la coordinación asumida por el Servicio de Documentación Multimedia http://campusvirtual.ucm.es/portal/laboratorio2.html (en cuyo ámbito nació e-DocuInfo/TVDoc) para el acceso de la facultad de ciencias de la información al campus virtual de la universidad complutense. Y en consecuencia, para su difusión a nivel nacional e internacional, merced a la amplia oferta de posibilidades de las plataformas planteadas para la docencia (semipresencial y virtual), investigación y producción multimedia. La puesta en marcha de canales de este tipo se justifica por la inexistencia de mediatecas en línea y de canales IPTV en el área de biblioteconomía y documentación, ni en el ámbito específico de la documentación informativa o de medios de comunicación social, independientemente de otros motivos expuestos a lo largo de estas páginas.
There is also mentioned several examples of contributed contents related to cinema, press, public advertising and public relations and radio and television. Finally it is noted the different collaborations established

by the university and several companies for the continuous diffusion of the thematic channel as well as future overcomes.

\section{Introducción}

Este trabajo apuesta por la consolidación definitiva del canal temático RTVDoc, como plataforma web para la formación, investigación, recuperación y difusión de materiales didácticos multimedia en el ámbito de las ciencias de la información; y más concretamente de los archivos, servicios y centros de documentación de medios de comunicación social o medios informativos. Se destacan asimismo las aplicaciones de los contenidos del canal en internet en el área de la formación semipresencial y virtual aplicada al estudio de las ciencias informativas. Se parte de la no existencia en 2007 de un canal temático sobre documentación en medios de comunicación social, por lo que decidimos a finales de 2006 llenar ese vacío con la propuesta de establecimiento de un canal web con las características mencionadas. La hipótesis se 
fundamentó en la posibilidad real, puesto que se pusieron en marcha por entonces canales IPTV sobre temáticas diversas, de aplicación al ámbito de la documentación informativa.

\section{Objetivos}

Los objetivos se resumen en dos, fundamentalmente, aunque más adelante se especifican pormenorizadamente, así como las respuestas o logros a dichos objetivos: producción de contenidos dirigidos al ámbito docente e investigador, y su correspondiente consulta o recuperación y difusión informativas. Información científica basada en todo momento en la investigación, el debate y la intercomunicación en documentación informativa: documentación periodística, audiovisual y publicitaria y de relaciones pública, estructurada en los siguientes bloques temáticos: investigación, servicios y centros de documentación en medios de comunicación social, tecnología digital, formación, publicaciones y bases de datos, propiedad intelectual y derechos de autor. Que se vertebran, asimismo, en diversas secciones y ámbitos de estudio e investigación en los que los canales se especializan: título propio, investigaciones de alumnos, proyectos de investigación, for- mación semipresencial, formación virtual, libros electrónicos, sub-portales (cine, publicidad, publicaciones electrónicas en documentación), doctorado en línea, materiales didácticos multimedia producto de proyectos de innovación educativa desarrollados con anterioridad.

El segundo objetivo, propiamente tecnológico, está basado en canales temáticos IPTV (televisión web) en base a los recursos que proporciona Internet y las herramientas de hardware y software de que dispone el equipo de investigación en el que se sustenta el proyecto. En consecuencia: uso profuso de las posibilidades del portal y sus canales de producción y difusión de contenidos a (Videoteca, Fonoteca, Fototeca, Hemeroteca...), con posibilidad de demanda de información multimedia "a la carta”, e incluso con calidad broadcast, en función del servidor de streaming al que tiene acceso la plataforma propuesta.

\section{Metodología}

El trabajo canaliza los proyectos de investigación y difusión de la producción propia de la facultad de ciencias de la información de Madrid y de sus profesores, alumnos, investigadores y profesionales de los medios de comunicación social, independientemente de la extensión a otras facultades, universidades e instituciones especializadas 
nacionales e internacionales. Se trata en todo momento de producción propia o ajena con los correspondientes permisos de publicación y difusión informativa. Por otra parte, los documentos que conforman la mediateca de los canales son pertinentes y sometidos a una selección previa. La mues- tra documental presenta temáticas estrechamente relacionadas en todo momento con las ciencias de la información y aplicables como materiales didácticos multimedia para su utilización en la docencia e investigación en el área de conocimiento de las ciencias de la información.

\section{Antecedentes $y$ estado de la cuestión}

Previamente a la propuesta de establecimiento de los canales web, motivo de este trabajo, se han llevado a cabo otras actuaciones que suponen un claro antecedente a la actividad investigadora ahora propuesta, y que confluye en una mayor aportación y aplicaciones a la docencia, investigación y producción multimedia a través de un gran portal (portal de portales) y de la difusión en línea $\mathrm{y} / \mathrm{o}$ en diferido de contenidos multimedia y de programación en tiempo real vía canales temáticos IPTV-televisión vía internet. Como antecedentes pueden mencionarse los siguientes, producto de proyectos de investigación previos: 1 . Configuración y puesta en funcionamiento de portales diversos en el ámbito de las ciencias de la información; 2. Fuentes de información en línea-en tiempo real; 3. Propuesta de establecimiento de una "Red iberoamericana de documentación informativa"; 4. Desarrollo de nuevas metodologías docentes: producción de materiales docentes multimedia en línea; 5. Espacio de Coordinación de Centro (Facultad de CC. Información) para el acceso al campus virtual de la UCM para profesores y alumnos; 6. Biblioteca digital: recuperación de información distribuida; Fondo documental-filmográfico del director José Val del Omar: biblioteca digital-portal en internet. El estado de la cuestión presenta una panorámica que permite afirmar que desde 2007, con el aumento considerable del ancho de banda, se posibilitaba el acceso a contenidos "bajo demanda" con calidad suficiente para rivalizar con los servicios tradicionales de televisión: por entonces ya existían más de 216 millones de suscriptores a líneas fijas de banda ancha y más de 61 millones de suscriptores a líneas móviles del mismo tipo (a través de la telefonía de tercera generación). El incremento constante de conectividad a través de sistemas de Banda Ancha (ADSL, Cable MODEM, PLC...) para cientos de millones de usuarios de internet a nivel global, propiciaba un tráfico masivo de obras culturales en la red. Esta situación viene siendo contemplada por una serie de instituciones y empresas públicas y privadas con manifestaciones IP/TV (sistema de distribución de 
televisión y vídeo sobre IP) como, por ejemplo y entre otras muchas, YouTube, Googlevideo, Madridmas - Fundación para el conocimiento madri+d e Internautas TV, o la Universidad Politécnica de Valencia.

También el Dpto. de Biblioteconomía y Documentación (a través de su Servicio de Documentación Multimedia) y el Departamento de Derecho Constitucional de la Universidad Complutense han propuesto a través del Laboratorio Multimedia Complutense la puesta en marcha, en principio, de dos plataformas de televisión en línea o canales temáticos en ciencias de la información (TVDoc y E-TV) para emisión de programación en tiempo real (streaming) y en diferido ("video bajo demanda"), al mismo tiempo que funciona como archivo multimedia-mediateca en línea ("a la carta”). Las posibilidades de producción propia, emisión, recuperación y difusión son ilimitadas: la programación de dicho canal está abierta a un abanico muy amplio de actividades docentes y de investigación como seminarios, mesas redondas, defensa de tesis doctorales, entrevistas, videoconferencias, formación virtual y sus aplicaciones en programas de doctorado (como por ejemplo el Doctorado Iberoamericano presencial y en línea en Biblioteconomía y Documentación), el Máster de Bibliotecas (en la Facultad de Ciencias de la Documentación) y los POP de Periodismo y de Comunicación Social (en la Facultad de Ciencias de la Información), y un larguísimo etcétera aplicable a nivel nacional e internacional (piénsese en el Espacio Europeo e Iberoamérica). En suma, afrontamos el último desafío de la red, para los medios y para la Universidad, que es el uso del vídeo, de la "televisión a la carta", como embrión de la televisión del futuro, y como herramienta esencial para el e-Learning. Mediante la creación de plataformas y canales de video "a la carta" en un entorno universitario se obtiene también alcance universal debido a la difusión vía Internet: se trata en definitiva de la nueva generación de sitios web en Internet, de eminente contenido de información multimedia, con aplicaciones específicas.

\section{Proyectos de investigación: valor estratégico y relevancia}

La temática planteada con motivo de esta colaboración para Icono14, responde en realidad a la apuesta relacionada con proyectos de investigación llevados a cabo en el ámbito del Servicio de Documentación
Multimedia, ubicado en el marco de la Sección Departamental de Biblioteconomía y Documentación de la Universidad Complutense, y que se materializan en los portales y canales web temáticos aquí referen- 
ciados. Por ello, y en concordancia con los resultados investigadores anteriores, se pretenden obtener los siguientes resultados específicos:

1. Actualizar permanentemente los contenidos multimedia que genera el portal de portales o portal de documentación informativa e-Docuinfo, estructurado en secciones diversas sobre documentación en ciencias de la información, así como los contenidos asociados a los canales web citados en el resumen.

2. Emitir en tiempo real o en diferido ("vídeo bajo demanda", en realidad "multimedia bajo demanda") dichos materiales a través de las plataformas temáticas IPTV generadas al efecto: "TVDoc" y "E-TV" (Universidad Complutense)y "Cine@Tele Canal Video" de la empresa Grupo ExportFilm que lidera Antonio Carballo. Con esta actuación se propone la connivencia Universidad-Empresa. Los materiales presentan una estructura basada en metadatos convenientemente normalizados (Méndez: 2006).

3. La producción generada constituye una mediateca multimedia en línea (hemeroteca, fototeca, fonoteca, videoteca...).

4. Los materiales producidos y su recuperación y difusión vía web se aplicarán a la docencia-formación semipresencial y virtual y a la investigación en distintos ámbitos de trabajo, tanto universitario como empresarial.

5. Otro objetivo que se persigue es la mejora del diseño actual que en principio presentan los canales temáticos que hemos configurado: precisan de una apariencia más "amable" y usable, en la línea de la Mediateca de Madrid+d.

6. Todo lo apuntado supone un avance claro en la línea de investigación que venimos desarrollando en los últimos 8 años.

7. Utilización profusa del campus virtual de la universidad complutense, aprovechando la responsabilidad que ha recaído en el autor de estas páginas entre los años 2005 y 2009 como responsable del Espacio de Coordinación de Centro (Facultad de Ciencias de la Información) para el acceso al campus virtual de la Universidad Complutense.

8. En fin, difundir exhaustivamente los resultados de la investigación vía medios de comunicación y otras instituciones informativas especializadas.

El valor estratégico que sin duda presentan estas actuaciones y la relevancia que en consecuencia les caracteriza se resume en los siguientes puntos: A) Vehiculan el conocimiento que genera la facultad de ciencias de la información en sus diversos ámbitos en relación con los medios de medios de comunicación; B) El portal de documentación informativa y los canales temáticos configurados al efecto, como producto 
final de la investigación, se presenta como una verdadera "Comunidad virtual de usuarios de documentación informativa”, en la que el usuario real y potencial participa en todo momento con aportaciones y sugerencias. Es la filosofía que es estos momentos más que nunca caracteriza a la red de redes (se ha consolidado el concepto de red social o sociedad-red): conocimiento distribuido y colaborativo, que es precisamente la filosofía que subyace en la estructura de contenidos que presentan las plataformas web cuya implantación definitiva defendemos; C) Entre otras funciones, los portales-canales vehiculan, entre otras realizaciones y actuaciones, por ejemplo, los proyectos de investigación y difusión de la producción propia de la Facultad de Ciencias de la Información y de sus profe- sores, alumnos, investigadores y profesionales de los medios de comunicación social, independientemente de la extensión a otras facultades, universidades e instituciones especializadas nacionales e internacionales; D) En fin, el planteamiento aquí expresado contribuirá sin duda a la mentalización del uso inevitable de la red de redes y el campus virtual de la UCM, puesto que proporciona fondos documentales relevantes en la conformación de asignaturas e investigaciones, tanto presenciales como virtualizadas a través del mencionado Espacio de Coordinación de Centro, con el que los portales y canales mantienen una muy estrecha colaboración, pudiendo abrir diversidad de vías de difusión a través de la red de redes.

\section{Logros obtenidos en relación con objetivos pormenorizados propuestos}

\subsection{Objetivo 1}

Actualizar permanentemente los contenidos multimedia que genera el portal de portales o portal de documentación informativa e-Docuinfo, estructurado en secciones diversas sobre documentación en ciencias de la información, así como los contenidos asociados a los canales web citados en el resumen. (RTVDoc, E-TV, Cine@Tele Online).

Logros: algunos ejemplos de actuaciones relacionadas con objetivo 1 . http: / / multidoc.rediris.es/edocuinfo/ (Portal de portales eDocuinfo, con canal web RTVDoc integrado y posibilidad de emisión vía internet en tiempo real) (López Yepes: 2007b).

http://multidoc.rediris.es/edocuinfo/ind ex.php?option $=_{\text {com }}$ content $\&$ task $={ }_{\text {sectio }}$ $\underline{\text { n\&id }=2 \& \text { Itemid }=28} \quad$ (Documentación cinematográfica, con portal Cinedocnet integrado) 
http:/ / complumedia.ucm.es/canal.php?p $1=$ rtvdoc (Contenidos accesibles a través del canal docente Complumedia-RTVDoc)

\subsection{Objetivo 2:}

Emitir en tiempo real o en diferido ("vídeo bajo demanda", en realidad "multimedia bajo demanda") dichos materiales a través de las plataformas temáticas IPTV (resultado de este proyecto en su segunda fase) generadas al efecto: TVDoc y E-TV (Universidad Complutense) y Cine@Tele Online de la empresa Grupo ExportFilm que lidera Antonio Carballo. Con esta actuación se propone la connivencia UniversidadEmpresa (Hernández, Pozuelo, López Yepes, Cámara: 2009) (López Yepes, Cámara: 2009).

Logros: TVDoc ha pasado a denominarse RTVDoc a tenor de la programación radiofónica iniciada:

http://www.ucm.es/info/tvdoc/radiodoc .php (Fonoteca del canal)

http://videoma.sim.ucm.es/videoma/wm /wmv baja yepes/320476/14796 1 14 6 videos 1368 WM BAJA.wmv (Primer programa de radio: emisión en tiempo real).

\subsection{Objetivo 3}

La producción generada constituye una mediateca multimedia en línea (hemeroteca, fototeca, fonoteca, videoteca...).

Logros: Página de bienvenida de la mediateca: http://www.ucm.es/info/tvdoc/

\subsection{Objetivo 4}

Los materiales producidos y su recuperación y difusión vía web se aplicarán a la docencia-formación semipresencial y virtual y a la investigación en distintos ámbitos de trabajo, tanto universitario como empresarial.

Logros: Aplicación a Primer Ciclo, Tercer Ciclo, Postgrado, Títulos propios, así como en el ámbito de Seminarios, Congresos, Jornadas... a través del Espacio de Coordinación de Centro (ECC) para el acceso al campus virtual por parte de profesores y alumnos (más de 7.500 personas) (el director del proyecto ha sido coordinador de dicho acceso, en representación del Dpto. de Biblioteconomía y Documentación, hasta julio de 2009, tras cinco cursos académicos).

http://www.ucm.es/pags.php?tp=Titulac iones\&a $=$ estudios\&d $=$ muestratitulacion. php \&idt $=10$ (Primer ciclo Comunicación Audiovisual)

http://www.ucm.es/pags.php?tp=Titulac iones\&a $=$ estudios\&d $=$ muestratitulacion. php\&idt $=95$ (Primer ciclo Publicidad y Relaciones Públicas)

http://www.ucm.es/pags.php?tp=Titulac iones\&a $=$ estudios\&d $=$ muestratitulacion. php\&idt $=100$ (Primer ciclo Periodismo) http://www.ucm.es/pags.php?tp=M\%E1 steres $\% 20$ Oficiales\&a $=$ estudios\&d= muestramaster.php\&idm $=61$ (Máster en Periodismo) 
http: / / www.ucm.es/pags.php?tp=M\%E1 steres $\% 20$ Oficiales\&a $=$ estudios\&d= muestramaster.php\&idm=62 (Máster en Comunicación Social)

http: / / www.ucm.es/centros/webs/ebibli o/index.php?tp=Másteres $\% 20$ Oficiales\&a Eestudios\&d=muestramaster.php\&idm $=6$ 3 (Máster en Gestión de la Documentación y Bibliotecas)

http: / / www.ucm.es/centros/webs/ebibli o/pags.php?tp=Estudios $\% 20 \mathrm{de} \% 20$ Doctor ado\%20-\%20Facultad \%20de\%20Bellas\% 20Artes\&a $=$ estudios $\& \mathrm{~d}=$ muestradoctorad o.php\&doct $=6$ Doctorado en Documentación: Fundamentos, Tecnologías y Aplicaciones (Doctorado en Ciencias de la Documentación)

http: / / www.ucm.es/centros/webs/fcinf /pags.php?tp=Estudios $\% 20 \mathrm{de} \% 20$ Doctora do\%20-\%20Facultad \%20de\%20Bellas \%20 Artes\&a $=$ estudios $\& \mathrm{~d}=$ muestradoctorado .php\&doct $=13$ Doctorado en Teoría, Análisis y Documentación Cinematográfica (López Yepes, López Yepes, J.: 2009). http: / / www.ucm.es/pags.php?tp=Servici os de Apoyo\&a $={ }_{\text {centros } \& d=\text { entidad- }}$ 2479.php Título propio Experto en Información y Documentación Ambiental (Canal web RTVDoc: sitio en directorio homepage Facultad Ciencias de la Información)

\subsection{Objetivo 5}

Otro objetivo que se persigue es la mejora del diseño actual que en principio presen- tan los canales temáticos que hemos configurado: precisan de una apariencia más “amable" y usable, en la línea de la Mediateca de Madrid+d.

Logros: La versión utilizada en esta primera fase del proyecto corresponde a Joomla 1.0. En la segunda fase del proyecto se trabajará con Joomla versión 1.5 :

http://multidoc.rediris.es/edocuinfo/ad ministrator/index.php

\subsection{Objetivo 6}

Todo lo apuntado supone un avance claro en la línea de investigación que venimos desarrollando en los últimos 8 años (véase proyectos de investigación previos aludidos y la bibliografía seleccionada).

Logros: Propuesta de establecimiento para la segunda fase del proyecto de una "Red iberoamericana de documentación informativa" Infodocnet, en la que se integran los portales y canales establecidos (López Yepes: 2005, 2008a):

http://multidoc.rediris.es/infodocnet/ad ministrator/

\subsection{Objetivo 7}

Utilización profusa del campus virtual de la universidad complutense, aprovechando la responsabilidad que recae en el autor de estas páginas como responsable del Espacio de Coordinación de Centro (Facultad de Ciencias de la Información) para el acceso al campus virtual de la Universidad Complutense (hasta julio de 2009, tras cinco 
curso académicos consecutivos) (López Yepes: 2007c).

Logros: Campus virtual UCM y presentación del canal RTVDoc en el marco de la V Jornada del Campus virtual-UCM, febrero 2009 (López Yepes: 2008b): https: / / www.ucm.es/campusvirtual/CV UCM/index.php (sitio campus virtualUCM)

http://www.ucm.es/info/tvdoc (Presentación del canal RTVDoc a cargo del autor: V Jornada Campus Virtual UCM, febrero 2009) (Acceso al documento tecleando en campo "Descripción": taller multimedia)

\subsection{Objetivo 8}

En fin, difundir exhaustivamente los resultados de la investigación vía medios de comunicación y otras instituciones informativas especializadas.

Logros: RTVDoc ha pasado a denominarse Complumedia-RTVDoc para mayor difusión vía Universidad Complutense: dispone en estos momentos de una configuración oficial como canal docente institucional:

Complumedia-RTVDoc:

http: / / complumedia.ucm.es/canal.php?p $1=$ rtvdoc lo que sin duda supone una mayor y mejor difusión informativa.

\section{Relación de ámbitos docentes y de investigación, $y$ foros de publicación $y$ difusión informativas}

Con sus enlaces correspondientes, en los que han estado presentes a lo largo -a título de ejemplo- de 2009 (en algunos casos, que se especifican, también con extensión a 2010) las temáticas planteadas con motivo de este artículo (e-Docuinfo, TVDoc/RTVDoc, E-TV, Cine@Tele Online), se relacionan a continuación las actuaciones y realizaciones llevadas a cabo en el marco de dichas temáticas:

- Título propio Universidad Complutense, "Experto en Información y Documentación ambiental” (2008-2009).

La presencia del medio ambiente en los medios de comunicación. En Manual de experto en información y documentación ambiental. Madrid, ANABAD (En prensa).

Máster en Documentación Digital: Máster y curso de postgrado online en Documentación Digital. Universidad Pompeu Fabra (2008-2009 y 20092010).

Documentación cinematográfica en línea. Documentación Digital. Master y curso de postgrado Online en Documentación Digital (11 a edición 2008/9) [en línea]. En Cristòfol Rovira; Lluís Codina (dir.). Documentación digital. Barcelona: Área de Ciencias de la Documentación. Departamento de Periodismo y de Co- 
municación Audiovisual. Universidad Pompeu 2009. http: / /www.documentaciondigital.org ISBN 84-88042-39-6 (López Yepes: 2006-2009).

- Primer Ciclo: Documentación informativa-Sección Comunicación audiovisual: producción investigaciones alumnos publicadas en RTVDoc (2009) (López Yepes: 2006).

Dos ejemplos:

Redes sociales en internet. http: / / videoma.sim.ucm.es/videoma/ wm/wmv baja yepes/320476/147 9 6 1 146 videos 1369 WM BAJA.w mv (Campo de Luna, Camps: 2008).

Buscadores

(2008).

http://videoma.sim.ucm.es/videoma/ wm/wmv baja_yepes/320476/147 9 61146 videos 1463 WM BAJA.w mv (Blázquez, Díaz: 2008).

- Máster de Periodismo-Facultad de Ciencias de la Información (2008-2009 y 2009-2010).

- Máster de Comunicación SocialFacultad de Ciencias de la Información (2008-2009 y 2009-2010).

- Máster Gestión de Documentación y Bibliotecas-Facultad de Ciencias de la Documentación (2008-2009 y 2009. 2010).

- Doctorado en línea Documentación: Fundamentos, Tecnologías y Aplicacio- nes (2008-2009 y 2009-2010) (López Yepes, 2007a).

- VI Seminario hispano-mexicano de Bibioteconomía y Documentación (2009).

- Seminarios IV-VI Boletín ANABAD (En prensa) y Videografía 2007 a 2009 (en I Congreso Internacional Latina Sociedad de la Información-La Laguna http: / / www.revistalatinacs.org/09/So ciedad/actas/97yepes.pdf

Revista venezolana Enl@ce (2009).

Cine español en Internet para la docencia, investigación y producción: canal web-mediateca en línea RTVDoc (López Yepes: 2009a).

http:/ / dialnet.unirioja.es/servlet/lista articulos?tipo busqueda=ANUALIDA

D\&revista busqueda $=7671 \&$ clave bus queda $=2009$

http://dialnet.unirioja.es/servlet/artic ulo? codigo $=2932258$

Jornadas IBERSID: Avances y perspectivas en sistemas de información y documentación (2009).

Canales web-mediatecas en línea como instrumentos de producción, recuperación y difusión en ciencias de la información. Channels web-mediathecas on line for Information science production, research and diffusion. En García Marco, Francisco Javier (ed.). Zaragoza, Prensas Universitarias, 2009, 326 
págs. ISBN 978-84-92774-41-8. http://www.ibersid.org/

- Diplomado de Documentación SonoraFonoteca Nacional de México (2009).

Hacia un reaprovechamiento educativo y cultural de un archivo sonoro. Hacia una Red Mexicana de Archivos Sonoros REDMAS (Módulo VI).

http://www.fonotecanacional.gob.mx /capacitacion.html

http://www.servimx.com/fonoteca

- Jornada profesional SEDIC-Ministerio de Cultura: Fondos invisibles: los archivos audiovisuales que no conocemos (2009).

El damero audiovisual: archivos de particulares, instituciones y empresas.

http: / / www. sedic.es/actividadesjornada-fondos-invisibles-sept09.asp

- X Encuentro Internacional Virtual Educa 2009-Buenos Aires (Argentina) noviembre 2009.

Enseñanza a distancia, canales web universitarios y gestión de la información digital en medios de comunicación social: Complumedia-RTVDoc, E-TV, Cine@Tele Online.

http://www.virtualeduca.info/index. php?option $=_{\text {Com }}$ wrapper\&view ${ }_{\text {wra }}$ pper\&Itemid $=221$

http: / / www.virtualeduca.info/ponenc ias2009/484/ponenciaalfonsolopezyep es.doc
- I Congreso Internacional Sociedad Digital 26-28 octubre 2009 (Madrid)

La sociedad digital que informa y publica. Relación mcs, comunicación interactiva, producción internautas: Canales web-mediatecas en línea como instrumentos de producción, recuperación y difusión en ciencias de la información... a propósito de ComplumediaRTVDoc (próximo acceso electrónico a la ponencia presentada)

http: / / www.gruposocmedia.es/congr esosociedaddigital/

- Seminario de Documentación: Fundamentos, Tecnologías y Aplicaciones. Universidad Nacional de Costa Rica. Facultad de Filosofía y Letras. Escuela de Bibliotecología, Documentación e Información (2009)

Red multimedia de documentación informativa: gestión de información digital en medios de comunicación (enseñanza, investigación, producción multimedia).

http://www.una.ac.cr/bibliotecologia http://www.ebdi.una.ac.cr/seminario /index.htm

I Congreso Internacional Latina Sociedad de la Información (La Laguna) 9-11 diciembre 2009.

Canales web en ciencias de la información: documentación informativa $\mathrm{y}$ producción multimedia en Complumedia-RTVDoc 
http: / / www.revistalatinacs.org/09/So ciedad/convocatoria congreso.html

http://www.revistalatinacs.org/09/So ciedad/00 indexBBB.html

http://www.revistalatinacs.org/09/So ciedad/actas/97yepes.pdf

Grabación íntegra defensa cuatro tesis doctorales en el marco del Programa de Doctorado del Dpto. de Biblioteconomía y Documentación Universidad Complutense y la Escuela de Ciencias de la Información de la Universidad Autónoma de San Luis Potosí-México (2009). Acceso a la defensa íntegra de la investigación a través del canal Complumedia-RTVDoc, como otro de los contenidos generados con motivo del proyecto:

García Lagunas, Juan René. “Modelo de redes de bibliotecas universitaras para los estados del centro de México". http: / / complumedia.ucm.es/visor.ph p?video $=$ p87509kFU9JVg9RvjB4Tww (01:54:30).

Rodríguez Sierra, Beatriz. "La industria editorial en México: su evolución y participación en el desarrollo de colecciones de bibliotecas”.

http: / / complumedia.ucm.es/visor.ph p?video=YfrVazsVZteMY6zBeP8UnQ (01:43:58)

Ramos Fandiño, Guadalupe Patricia: orígenes, evolución y organización de los fondos" http: / / complumedia.ucm.es/visor.ph p?video=KGar6XNWnvsNkjCMZOEH $\underline{7 w}(01: 26: 58)$

Chacón Escobar, Rosa Elba. "Fondos bibliográficos antiguos de la Universidad de Ciencias y Artes de Chiapas, México: estudio histórico y descriptivo (siglos XVI-XVIII)".

http: / / complumedia.ucm.es/visor.ph p?video $=$ LTJtlwmmnMYvuCT1a2uVA g $(01: 29: 53)$

\subsection{Otras \\ actuaciones: ámbito e-television}

- Tendencias sociedad de la información http: / / etelevision.es/index.php?optio $\underline{\mathrm{n}}={ }_{\text {com content } \& \text { view }}={ }_{\text {category } \& \mathrm{id}}=$ 29:tendencias\&layout $=$ default

- Derecho de los Medios y Contenidos On Line

http: / / etelevision.es/index.php?optio $\underline{\mathrm{n}}={ }_{\text {com }}$ content $\&$ view $={ }_{\text {category } \& i d}=$ 24: derecho-de-los-medios-ycontenidos-on-line\&layout $=$ default

- Políticas audiovisuales

http: / / etelevision.es/index.php?optio $\underline{\mathrm{n}}={ }_{\mathrm{com}}$ content $\&$ view $={ }_{\text {category } \& \mathrm{id}}=$ 23:polcas-audiovisuales\&layout= $\underline{\text { default }}$

- Media Literacy-Alfabetización digital http: / / etelevision.es/index.php?optio $\underline{\mathrm{n}} \overline{\mathrm{Com}}_{\mathrm{conten}} \&$ view ${ }_{\text {category } \& \mathrm{id}}=$ 
22:media-literacy-

alfabetizaciigital\&layout $=$ default

- Canales de Video para apoyo docente que se utilizan como material en clase y en grupos de estudio.

http: / / e-television.es/canales /

- Congreso Internacionales de Ética y Derecho de la Información. VII Edición en Valencia, 13 y 14 de noviembre de 2009.

http: / / fundacioncoso.org/ congreso/6 ciedi/index.htm

- Participación en el Grupo Red Derechotics, de la Universidad de Valencia: Red de especialistas en Derecho de las Tecnologías de la Información y Comunicación.

http://www.derechotics.com/

\subsection{E-tv: canales}

Teleclip TV forma parte de los canales temáticos de e-Televisión, consecuencia de investigaciones previas desarrolladas en el marco del Catálogo de Transferencia de Tecnología de OTRI-UCM y del sistema $\mathrm{mi}+\mathrm{d}$ de la Comunidad de Madrid.

e-Televisión trabaja en el desarrollo de Teleclip TV en estrecha relación con un equipo de producción de la Universidad de A Coruña, así como con varios Partners tecnológicos y del ámbito educativo para el desarrollo del Canal en España, Iberoamérica y EEUU.

http: / / www.teleclip.tv/
Periodistas en el Cine es un Canal que publica las prácticas para alumnos de Derecho de la Información, de la Licenciatura de Periodismo.

Se analizan fragmentos comentados de películas en los que surgen dilemas éticos. Ausencia de Malicia, Buenas Noches y buena Suerte, El Dilema, El año que vivimos peligrosamente, Quiz Show o Verónica Guerin, entre otros. Es un canal respetuoso con los derechos de autores y productores, elaborado como ejercicio del derecho de cita y de uso en la investigación.

Incluye breves cortes de video para debate en clase, para discusión en foros, para el aprendizaje del "proceso ético en las redacciones de noticias".

Materias que pueden localizarse en el buscador: derecho de rectificación, ética personal, secreto profesional, fuentes informativas, falsedad, independencia, etc.

http: / / e-television.es/index.php?option= com content\&view $=$ article $\& i d=16 \&$

$\underline{\text { Itemid }=83}$

Análisis ético y estético es un canal en el que se analizan fragmentos comentados de películas en los que surgen dilemas éticos en obras como Casablanca, La infancia de Iván, Psicosis, Saw, Los siete samuráis, Bailar en la oscuridad, etc. En la descripción del video se analizan los elementos esenciales de la secuencia elegida.

Es un canal respetuoso con los derechos de autores y productores, elaborado como 
ejercicio del derecho de cita y de uso en la investigación; los cortes no suelen superar los 5 ó 6 minutos.

http: / / e-television.es/index.php? option $={ }_{\text {com content } \& \text { view }}=$ article\&id $=$ $\underline{17 \& \text { Itemid }=84}$

\subsection{Próximas} actuaciones (2010)

Jornadas sobre IPTV y VOD 2010 (Febrero 2010): por qué y para qué unas Jornadas sobre IPTV y Educación

La IPTV fue conocida por el público general en 2006-2007 cuando el fenómeno YouTube alcanzó millones de entradas, razón por la que Google lo incorporó a su conglomerado de Internet. También porque desde 2005 las operadoras de telecomunicaciones ofrecen contenidos de televisión a través de redes de Internet (Imagenio, Jazztel TV) o porque los sitios y web clásicos han ido introduciendo vídeo, emitiendo en directo programas. No es sólo una revolución en el sector de los medios, o de las "teleco", sino en información, entretenimiento $y$, sobre todo, en educación y formación. Según el informe reciente del Pew Internet Project (julio 2009), los usuarios de sitios de video, ya sea en streaming ya en descargas (o sitios de compartir) llega al 63\% de adultos en 2009, lo que significa casi el doble desde el 2006. También nos llegan datos sobre cómo los usuarios de móviles están utilizando el "celular" para acceder a Internet y para ver vídeos. El informe de Nielsen manifiesta que el decrecimiento de espectadores de televisión es significativo, no en cuanto contenidos, sino en cuanto modo de uso. Se ve menos "tele" en casa (un 4\%) y más en Internet (9\%).

En estas Jornadas se analizarán las tendencias y oportunidades de las INTERNET TV (tanto la TVIP como el Video on Demand -VOD-) para Universidades, Centros de Investigación y Formación, Empresas orientadas a las TICs, así como para colegios e institutos, dedicando algunas sesiones a los profesores como docentes y "prescriptores" de las tecnologías en las aulas.

http: / / etelevision.es/index.php?option ${ }_{C}$ om content \&view $=$ frontpage $\&$ Itemid $=1 \&$ $\underline{\text { Itemid }}=1 \&$ Itemid $=1$

\subsection{Otras actuaciones: ámbito cineetele online}

Estructura y sistematización de contenidos del canal IPTV Cine@Tele Online: http: / / www.cineytele.com/vervideo.php $? \mathrm{v}=\mathrm{CtvxYYmwCFw}($ Canal IPTV)

- Interrelación de contenidos con TVDoc/Complumedia-RTVDoc:

https: / / campusvirtual.ucm.es/SCRIP T/portal-50186124-3/scripts/serve home (TVDoc-RTVDoc)

- Directorio institucional: producción multimedia en línea. http://www. cineytele.com/inforegional2.php (Información institucional) 
- Directorio Cine: http://www.ciney tele.com/directorio.php?sec $=$ cine

- Directorio Televisión: http://www. cineytele.com/directorio.php?sec=tele

\subsection{Otros resultados añadidos}

Actuaciones en relación con la propuesta colaboración Universidad-Empresa. Algunos ejemplos:

- BARATZ. Gestionando el conocimiento: Colaboración en la puesta en marcha del Laboratorio multimedia de formación en línea, ubicado en el Dpto. de Biblioteconomía y Documentación http://www.baratz.es/ (Baratz) https: / / campusvirtual.ucm.es/SCRIP T/portal-50186124-2/scripts/serve home (Laboratorio multimedia: acceder a "Formación e investigación en línea").

- SEDIC-MINISTERIO DE CULTURA SOBRE ARCHIVOS AUDIOVISUALES: Jornada profesional.

Difusión informativa sobre las temáticas planteadas y desarrolladas en el proyecto de investigación (ya comentado anteriormente con más detalle).

http: / / www.sedic.es/actividadesjornada-fondos-invisibles-sept09.asp

\section{Conclusiones}

Este trabajo apuesta por la consolidación definitiva del canal temático ComplumediaRTVDoc, como plataforma web para la formación, investigación, recuperación y difusión de materiales didácticos multimedia en el ámbito de las ciencias de la información; y más concretamente de los archivos, servicios y centros de documentación de medios de comunicación social o medios informativos, concluyendo con los siguientes puntos:

1. Se consolida el establecimiento de canales WebRTV en el marco de la web social, y más concretamente, en nuestro caso, en el ámbito de la documentación informativa con la puesta en marcha de- finitiva del canal web-mediateca RTVDoc;

2. La actualización permanente y la proyección colaborativa y distribuida de sus contenidos, así como la difusión de los mismos a nivel ciberespacial;

3. La plataforma propuesta supone una programación permanente en tiempo real y en diferido, en forma de mediateca: hemeroteca, fonoteca, fototeca, videoteca;

4. El concepto "vídeo bajo demanda" ha evolucionado, en consecuencia, hacia el establecimiento de un nuevo concepto: "multimedia bajo demanda". 
5. Debe destacarse la existencia de las denominadas redes sociales científicas, cuyo protagonismo es muy importante en el marco de la red-social/sociedadred en la que nos encontramos.

6. Asimismo los denominados map-sites, mapas conceptuales o diagramas, cuya configuración permite sistematizar más inteligentemente los contenidos produ- cidos para una recuperación y difusión más sofisticadas.

7. Los canales web contribuyen, con su integración en la misma, a la expansión informativa de la "Red iberoamericana de documentación informativa”, con el desarrollo permanente de contenidos interrelacionados, lo que contribuye a un conocimiento global en el ámbito iberoamericano.

\section{Referencias}

BLÁZQUEZ, M. y DÍAZ, Es. (2008) Buscadores. RTVDoc. Madrid, Servicio de Documentación Multimedia, Facultad de Ciencias de la Información, Universidad Complutense, 17 de diciembre de 2008, 41 minutos (vídeo formato .wmv)

http://videoma.sim.ucm.es/videoma/wm/wmv b aja yepes/320476/147 $96 \quad 1 \quad 146$ videos 1463 WM BAJA.wmv (consulta 05.12.2009).

CAMPO DE LUNA, L. y CAMPS I. (2008). Redes sociales. RTVDoc. Madrid, Servicio de

Documentación Multimedia, Facultad de Ciencias de la Información, Universidad Complutense, 3 de diciembre de 2008, 66 minutos (vídeo formato .wmv) http: / /videoma.sim.ucm.es/videoma /wm/wmv baja yepes/320476/147 $96 \quad 1 \quad 146 \quad$ v ideos 1369 WM BAJA.wmv (consulta 05.12.2009).

HERNÁNDEZ PASCUAL, A.; POZUELO SANZ, A. J.; LÓPEZ YEPES, A. y Cámara Bados, V. (2009). Integración de contenidos multimedia en el campus virtual. V Jornada Campus Virtual UCM, Taller. RTVDoc. Madrid, Servicio de Documentación Multimedia, Facultad de Ciencias de la Información, Universidad Complutense, 10 de febrero de 2009, 115 minutos (vídeo formato .wmv) http: / /videoma .sim.ucm.es/videoma/wm/01 WM Baja UCM General/147 96 1_146_videos 1498 WM BAJA .wmv (consulta 05.12.2009).
LÓPEZ YEPES, A. (2005). Red iberoamericana de documentación multimedia-nodos español y mexicano: enseñanza a distancia, bibliotecas digitales, portales. En Actas de Virtual Educa 2005. Ciudad de México, mayo 2005 (CD-ROM).

--- (2006). Enseñanza multimedia en Documentación informativa: cursos y materiales didácticos en línea (Metodologías y herramientas para el desarrollo de materiales didácticos en el CV. Proyectos de innovación educativa aplicados al CV). En III Jornadas Campus Virtual UCM, 26 septiembre 2006. Madrid, Vicerrectorado de Innovación y Espacio Europeo de Educación Superior. Unidad de Apoyo Técnico y Docente al Campus Virtual UCM (UATD-CV), 2006.

--- (2006-2009) Documentación cinematográfica en línea [en línea].En Cristòfol Rovira; Lluis Codina (dir.).: Documentación digital. Barcelona: Grupo de investigación DigiDoc. Instituto Universitario de Lingüística Aplicada. Departamento de Periodismo y de Comunicación Audiovisual. Universidad Pompeu Fabra, 2006-2009. http: / / www.documentacion digital.org (consultado 05.12.2009).

--- (2007a). Gestión de información digital en la empresa audiovisual (cine y televisión: formación, investigación, producción multimedia) (Doctorado iberoamericano en línea: "Documentación: Fundamentos, Tecnologías y Aplicaciones"). En Memorias del XV Encuentro Internacional de Educación a Distancia, Guadalajara (México), 27 noviembre -1 diciembre 2006. México 
(Jalisco), Universidad de Guadalajara-Sistema de Educación Virtual, 2007 (CD-ROM).

--- (2007b). e-DocuInfo: un portal de documentación informativa para la formación, investigación y producción multimedia en línea. IV Seminario hispano-mexicano de investigación en Bibliotecología y Documentación). Boletín de la ANABAD, LVII (2007) núm.2.

--- (2007c). Formación semipresencial y virtual en Documentación Informativa en el ámbito del Departamento de Biblioteconomía y Documentación de la Universidad Complutense (1998-2007). Canales de Documentación. Revista de Biblioteconomía y Documentación, Vol.10, 2007 (http: / / www.um.es/ fccd/anales/ad10/ad1014.pdf (consulta 05.12.2009).

--- (2008a). La mediateca en el ámbito de la Red Iberoamericana de Documentación Informativa. En Memoria del I Seminario Hispano-Peruano de Bibliotecología y Documentación, Lima 23 a 26 de mayo de 2007. Lima, Biblioteca Nacional del Perú y Universidad Complutense-Dpto. de Biblioteconomía y Documentación, 2008, págs.81-93.

--- (2008b).Campus virtual y mediateca en línea: una experiencia docente innovadora en el ámbito de la documentación informativa. Actas del III Encuentro Ibérico de Docentes e Investigadores en Información y Documentación EDIBCIC 2008. Salamanca, EDIBCIC, 2008.

--- (2009). Cine español en Internet para la docencia, investigación y producción: canal web- mediateca en línea RTVDoc. Enl@ce: revista Venezolana de Información, Tecnología y Conocimiento, ISSN 1690-7515, Vol. 6, N . 1, 2009, págs.99-114

(http: / / dialnet.unirioja.es/servlet/articulo?codigo =2932258) (consulta 05.12.2009).

LÓPEZ YEPES, A. y CÁMARA BADOS, V. (2009). Integración de contenidos multimedia en el Campus Virtual de la Universidad Complutense: una experiencia concreta en el ámbito de las ciencias de la información a propósito del canal web-mediateca RTVDoc. En Actas de la V Jornada Campus Virtual Universidad-UCM, febrero 2009. Madrid, Universidad Complutense, 2009. (En prensa)

LÓPEZ YEPES, A. y LÓPEZ YEPES, J. (2009). El Programa de Doctorado 050 del Departamento de Biblioteconomía y Documentación de la Universidad Complutense y su proyección iberoamericana. En VI Seminario Hispano-Mexicano de Biblioteconomía y Documentación, Cáceres, Sigüenza, Madrid, 13 a 17 abril 2009. Boletín de la ANABAD, 2009 (En prensa).

MÉNDEZ RODRÍGUEZ, E. Ma (2006).Dublin Core, metadatos y vocabularios. En El Profesional de la Información, vol. 15, núm.2, marzo-abril 2006, p.84-86. http: / / elprofesionaldelainformacion. metapress.com/app/home/contribution.asp?referre $\underline{\mathrm{r}=\text { parent\&back to=issue }, 1,9 \text {; journal, 8, 53; } \text { homemai }}$ npublications, 1,1 (consulta 05.12.2009).

\section{Cita de este artículo}

LÓPEZ YEPES, A. (2010) Canales web en ciencias de la comunicación. Producción multimedia para la docencia e investigación en Documentación informativa. Revista Icono14 [en línea] 1 de Julio de 2010, Año 8, Vol. 2. pp. 164-181. Recuperado (Fecha de acceso), de http://www.icono14.net 